\title{
High-Resolution Direction-of-Arrival Estimation via Concentric Circular Arrays
}

\author{
Serdar Ozgur Ata and Cevdet Isik \\ Department of Electronics and Communications Engineering, Istanbul Technical University, Maslak 34469, Istanbul, Turkey \\ Correspondence should be addressed to Serdar Ozgur Ata; serdar.ozgur.ata@gmail.com
}

Received 5 February 2013; Accepted 28 February 2013

Academic Editors: Y.-S. Chen, L.-M. Cheng, C. S. Lin, and W. Zuo

Copyright (C) 2013 S. O. Ata and C. Isik. This is an open access article distributed under the Creative Commons Attribution License, which permits unrestricted use, distribution, and reproduction in any medium, provided the original work is properly cited.

\begin{abstract}
Estimating the direction of arrival (DOA) of source signals is an important research interest in application areas including radar, sonar, and wireless communications. In this paper, the problem of DOA estimation is addressed on concentric circular antenna arrays (CCA) in detail as an alternative to the well-known geometries of the uniform linear array (ULA) and uniform circular array (UCA). We define the steering matrix of the CCA geometry and investigate the performance analysis of the array in the DOAestimation problem by simulations that are realized through varying the parameters of signal-to-noise ratio, number of sensors, and resolution angle of sensor arrays by using the MUSIC (Multiple Signal Classification) algorithm. The results present that CCA geometries provide higher angle resolutions compared to UCA geometries and require less physical area for the same number of sensor elements. However, as a cost-increasing effect, higher computational power is needed to estimate the DOA of source signals in CCAs compared to ULAs.
\end{abstract}

\section{Introduction}

The problem of estimating the direction of arrival (DOA) of source signals by sensor arrays has been widely researched for applications such as radar, sonar, and wireless communication technologies. In radar applications as part of military systems, estimating DOAs of signals is crucial to differentiate targets, whereas in communications DOA information provides spatial diversity to increase the number of users communicating simultaneously [1].

In case of multisource signals, it is inevitable to employ sensor array configurations to estimate the DOA of each signal. Increasing the number of sensors in an array provides a higher signal-to-noise ratio (SNR) by processing the signals received from the sensors in parallel [2]. In DOA estimation applications the source signals are usually modeled as narrowband, or in case of wideband signals, and they may be processed as a composition of narrowband signals $[3,4]$.

There are various techniques in DOA estimation, most of them are either model-based or eigen-analysis-based ones. Model-based techniques, including least mean square (LMS) and sample matrix inversion based algorithms, may have higher computational complexity [5]. On the other hand, eigen-analysis-based techniques rely on the phase differences of signals impinging on array elements. One of these methods is the well-known MUSIC (Multiple Signal Classification) algorithm that depends on the signal subspace separation by using eigen-analysis techniques $[6,7]$. The algorithm has already been widely employed in uniform linear arrays (ULA) and uniform circular arrays (UCA).

Array geometries impose constraints in DOA estimations. The most important disadvantage of the ULA geometry is that it can only estimate the azimuth angle. To overcome this problem, UCAs have been employed in applications requiring the estimation of both azimuth and elevation angles [4]. Recently some optimization algorithms have been developed for UCA geometries in smart antenna systems [8, 9]. As an alternative geometry of sensor arrays, the concentric circular arrays (CCA) are proposed and investigated in this paper for DOA estimation. This way, we tried to achieve better angle resolutions compared to ULAs, and smaller physical areas compared to UCAs with the same number of array elements. CCAs have already been analyzed in terms of side-lobe reduction techniques in $[10,11]$.

The paper is organized as follows. Section 2 gives preliminary information on signal subspace decomposition in noisy 
environment and MUSIC algorithm based on the eigenanalysis approach. In Section 3, as an original contribution in the area, we describe the DOA estimation problem for CCAs and define the steering matrix of a CCA geometry. The performance analysis is examined through comparative simulations on several antenna geometries in Section 4. The role of various parameters is also analyzed in the estimation performance. The paper is concluded in Section 5 with future directions.

\section{Preliminaries}

2.1. Signal Subspaces. The observed signal vector $\mathbf{x}(t)$ on an antenna array of $M$ elements can be modeled as a linear combination of $P$ source signals $\mathbf{s}(t)$ with different DOAs under additive noise $\mathbf{n}(t)$ as

$$
\mathbf{x}(t)=\mathbf{A s}(t)+\mathbf{n}(t),
$$

where the steering matrix of the system $\mathbf{A}$ is a positivedefinite matrix of dimension $M \times P$ and includes the phase relationships of impinging signals depending on the geometry of the array. A can be represented as

$$
\mathbf{A}=\left[\mathbf{a}\left(\theta_{1}, \phi_{1}\right), \ldots, \mathbf{a}\left(\theta_{P}, \phi_{P}\right)\right]_{M \times P},
$$

where its $a_{i j}$ th element is related to the $i$ th element of the sensor array, the response of that element to the $j$ th source signal, and its distance to the reference point of the coordinate system.

The $M \times M$ correlation matrix $\mathbf{R}_{\mathbf{x x}}$ of the observed signal is helpful in estimating the subspaces of source signals and the noise to differentiate the source signals accordingly. Denoting the statistical expectation operator by $E[\cdot], \mathbf{R}_{\mathbf{x x}}$ is defined as

$$
\begin{aligned}
\mathbf{R}_{\mathbf{x x}} & =E\left[\mathbf{x}(t) \mathbf{x}^{H}(t)\right] \\
& =\mathbf{A} E\left[\mathbf{s}(t) \mathbf{s}^{H}(t)\right] \mathbf{A}^{H}+E\left[\mathbf{n}(t) \mathbf{n}^{H}(t)\right] .
\end{aligned}
$$

The noise variance of all elements of the array can be assumed as constant and equal to $\sigma^{2}$. In terms of noise characteristics, the array elements are assumed as independent among each other. Moreover, as the source signals are usually uncorrelated, the correlation matrix $\mathbf{R}_{\mathbf{s s}}$ of the source signal vector $\mathbf{s}(t)$ will be nonsingular and its rank is equal to $P$. Similarly, $\mathbf{A R}_{\mathbf{s s}} \mathbf{A}^{H}$ will have a dimension of $M \times M$ with a rank $P$ less than $M$. Then, the $M-P$ eigenvectors of the matrix will have zero eigenvalues; that is, for $M-P<i \leq M$ and $\mathbf{q}_{i}$ is an eigenvector of $\mathbf{R}_{\mathrm{ss}}$, then

$$
\mathbf{A R}_{\mathbf{s s}} \mathbf{A}^{H} \mathbf{q}_{i}=0
$$

is satisfied. If (4) is multiplied by $\mathbf{q}_{i}^{H}$ from left side,

$$
\left(\mathbf{A}^{H} \mathbf{q}_{i}\right)^{H} \mathbf{R}_{\mathrm{ss}}\left(\mathbf{A}^{H} \mathbf{q}_{i}\right)=0
$$

is attained. A direct consequence of (5) is that columns of the matrix $\mathbf{A}$ are orthogonal to $\mathbf{q}_{i}$ and therefore $\mathbf{A}^{H} \mathbf{q}_{i}=0$. As $\mathbf{R}_{\mathbf{s s}}$ does not exist in real applications, it is convenient to compute
$\mathbf{R}_{\mathbf{x x}}$ and by determining the eigenvectors of the correlation matrix of the observed signals, it's possible to estimate the $\mathbf{Q}$ matrix whose columns are the $\mathbf{q}_{i}$ vectors. On the other hand, for $1 \leq i<P$, the eigenvector $\mathbf{q}_{i}$ of $\mathbf{R}_{\mathbf{s s}}$ satisfies

$$
\mathbf{R}_{\mathbf{s s}} \mathbf{q}_{i}+\sigma^{2} \mathbf{I} \mathbf{q}_{i}=\left(\lambda_{i}+\sigma^{2}\right) \mathbf{q}_{i}
$$

It is possible to decompose $\mathbf{R}_{\mathbf{x x}}$ as $\mathbf{R}_{\mathbf{x x}}=\mathbf{Q}\left[\Lambda+\sigma^{2} \mathbf{I}\right] \mathbf{Q}^{H}$ where $\mathbf{Q}$ matrix is formed by the eigenvectors corresponding to the eigenvalues. The $\mathbf{Q}$ matrix can be decomposed into two different matrices $\mathbf{Q}_{s}$ and $\mathbf{Q}_{n}$, where $\mathbf{Q}_{s}$ is the matrix with columns of eigenvectors related to the $P$ source signals and $\mathbf{Q}_{n}$ is the matrix with columns of $M-P$ eigenvectors of noise eigenvalues of $\sigma^{2}$, respectively. As $\mathbf{Q}$ is an orthogonal matrix, then $\mathbf{Q}_{s} \perp \mathbf{Q}_{n}$; hence, the noise eigenvectors are orthogonal to the steering vectors of the source signals. In the decomposition of $\mathbf{R}_{\mathbf{x x}}$, the diagonal matrix is formed by considering the eigenvalue ordering as $\lambda_{1} \geq \lambda_{2} \geq \cdots \geq \lambda_{M} \geq$ 0 . This way, the eigenvector and the corresponding eigenvalue pairs can easily be separated to the ones corresponding to the signal and noise subspaces accordingly. In this case, $\mathbf{R}_{\mathbf{x x}}$ is expressed as

$$
\mathbf{R}_{\mathbf{x x}}=\mathbf{Q}_{s} \Lambda_{s} \mathbf{Q}_{s}^{H}+\mathbf{Q}_{n} \Lambda_{N} \mathbf{Q}_{n}^{H}
$$

and $\Lambda_{N}=\sigma^{2} \mathbf{I}$. As all of the noise eigenvectors are orthogonal to the subspace spanned by the steering vectors of $\mathbf{Q}$, then the columns of $\mathbf{Q}_{s}$ will span the subspace orthogonal to the noise subspace.

2.2. DOA Estimation by MUSIC Algorithm. As the noise eigenvectors spanning the noise subspace is orthogonal to the columns of the steering matrix $\mathbf{A}, \mathbf{Q}_{n}^{H} \mathbf{a}(\theta, \phi)=0$ is satisfied at exact DOAs of the source signals at $(\theta, \phi) \in$ $\left(\theta_{1}, \phi_{1}\right),\left(\theta_{2}, \phi_{2}\right), \ldots,\left(\theta_{P}, \phi_{P}\right)$ values. This expression constitutes the main idea of DOA estimation. The MUSIC spatial spectrum is defined as

$$
\mathbf{P}_{\text {MUSIC }}(\boldsymbol{\theta}, \phi)=\frac{\mathbf{a}^{H}(\theta, \phi) \mathbf{a}(\theta, \phi)}{\mathbf{a}^{H}(\theta, \phi) \mathbf{Q}_{n} \mathbf{Q}_{n}^{H} \mathbf{a}(\theta, \phi)}
$$

and at original $(\theta, \phi)$ values, the denominator will be equal to zero and consequently $\mathbf{P}_{\text {MUSIC }}$ goes to infinity, then the DOA estimation of $(\widehat{\theta}, \widehat{\phi})$ becomes

$$
(\widehat{\theta}, \widehat{\phi})=\underset{(\theta, \phi)}{\arg \max } \frac{\mathbf{a}^{H}(\theta, \phi) \mathbf{a}(\theta, \phi)}{\mathbf{a}^{H}(\theta, \phi) \mathbf{Q}_{n} \mathbf{Q}_{n}^{H} \mathbf{a}(\theta, \phi)} .
$$

\section{DOA Estimation Problem on a Concentric Circular Array Geometry}

To formulate the DOA estimation problem in a CCA geometry, we need to review the DOA estimation by UCA first. Then, we define the steering matrix of CCA in Section 3.2.

In DOA estimation by UCA, $M$ sensors are located as shown in Figure 1 and the elevation and azimuth angles of the impinging signal are denoted by $\theta \in[0, \pi / 2]$ and $\phi \epsilon$ $[0,2 \pi]$, respectively. The angle between the $m$ th sensor and 


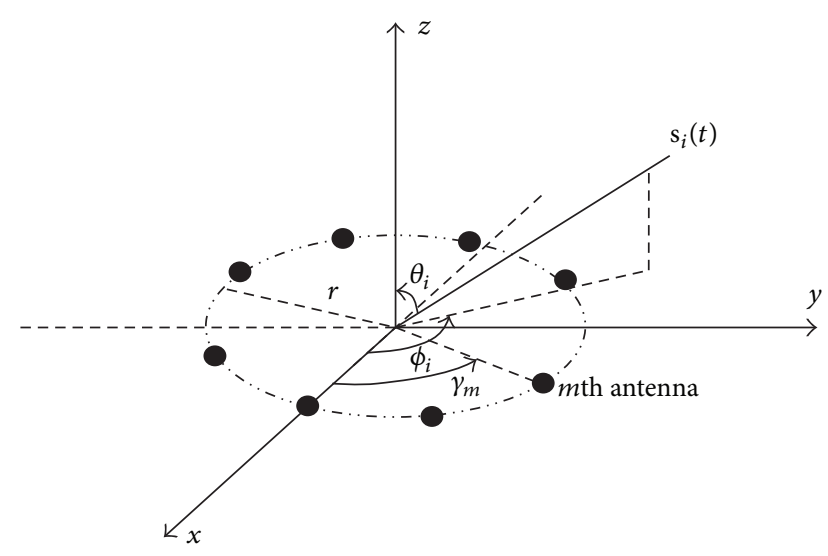

FIGURE 1: Uniform circular array geometry.

the $x$-axis of the coordinate system for $m \in[0, M-1]$ is represented by $\gamma_{m}=2 \pi(m / M)$. The position vector of each array element is then expressed as $\mathbf{r}_{\mathbf{m}}=\left[x_{m}, y_{m}, z_{m}\right]=$ $\left[r \sin \left(\phi_{m}\right), r \cos \left(\phi_{m}\right), 0\right]$, whereas the propagation directions of source signals are $\alpha_{\mathbf{i}}=(1 / c)\left[x_{i}, y_{i}, z_{i}\right]$ for all $i \in[0, P-1]$ $[12,13]$. The electric field measured by the $m$ th sensor of the array originating from the $i$ th source signal of a DOA $\left(\theta_{i}, \phi_{i}\right)$ is $E\left(\mathbf{r}_{\mathbf{m}}, t\right)=s_{i}(t) \exp \left(j \omega\left(t-\alpha_{\mathbf{i}} \cdot \mathbf{r}_{\mathbf{m}}\right)\right)$. Using the $\alpha_{\mathbf{i}}$ vector expressed in polar coordinates

$$
\alpha_{\mathbf{i}}=\frac{1}{c}\left(\sin \theta_{i} \cos \phi_{i}, \sin \theta_{i} \sin \phi_{i}, \cos \theta_{i}\right),
$$

then the phase difference of the $m$ th sensor element on the array according to the reference point becomes

$$
\begin{aligned}
\alpha_{\mathbf{i}} \cdot \mathbf{r}_{\mathbf{m}} & =\frac{1}{c}\left[\begin{array}{c}
\cos \phi_{i} \sin \theta_{i} \\
\sin \phi_{i} \sin \theta_{i} \\
\cos \theta_{i}
\end{array}\right] \cdot\left[\begin{array}{c}
r \cos \gamma_{m} \\
r \sin \gamma_{m} \\
0
\end{array}\right] \\
& =\frac{r}{c} \sin \theta_{i} \cos \left(\phi_{i}-\gamma_{m}\right) .
\end{aligned}
$$

The phase difference of the complex envelope of the field at $m$ th sensor with respect to the origin is then

$$
\Delta \phi_{m, i}=\omega\left(\alpha_{\mathbf{i}} \cdot \mathbf{r}_{\mathbf{m}}\right)=\frac{2 \pi}{\lambda} \sin \theta_{i} \cos \left(\phi_{i}-\gamma_{m}\right) .
$$

3.1. Concentric Circular Arrays. CCA geometry is constructed by using concentric UCAs as in Figure 2. In the figure, there are $H$ circular arrays and each array has $K_{h}$ sensor elements, where index $h$ denotes the number of the circles $1 \leq h \leq H$. The circular arrays may have different number of elements; therefore, they form different UCA geometries. When the centers of the circles are assumed to be the origin of the coordinate system, then the position of the $k_{h}$ th element on the $h$ th circle is

$$
\mathbf{r}_{h}=\left(r_{h} \cos \phi_{k_{h}}, r_{h} \sin \phi_{k_{h}}, 0\right) \text {. }
$$

In this array structure, $r_{h}$ is the radius of the $h$ th circle and the angle between two neighboring elements is

$$
\phi_{k_{h}}=2 \pi \frac{k_{h}}{K_{h}}
$$

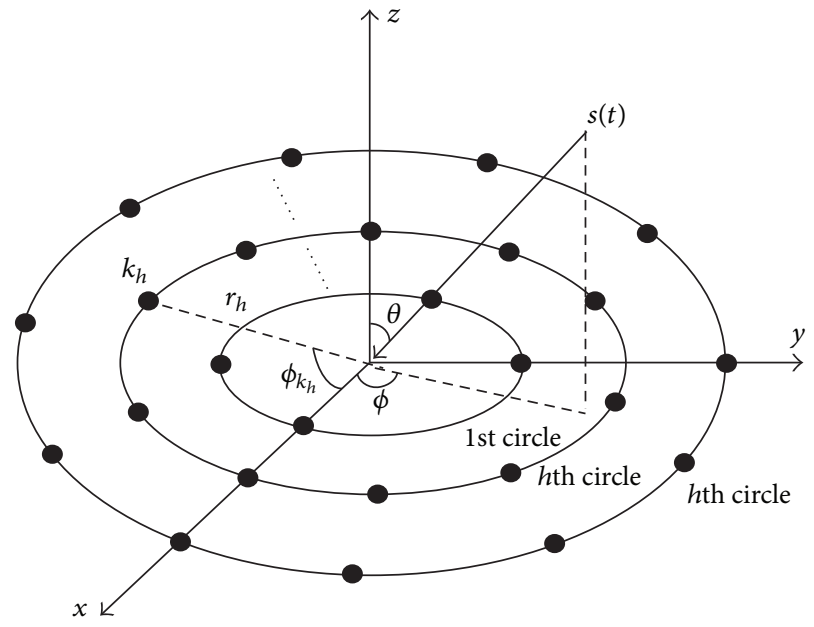

Figure 2: Concentric Circular Array geometry.

for $k_{h}=0,1, \ldots, K_{h}-1$, and $h=1,2, \ldots, H$. Applying (13) and (14) to (11) and (12), the phase component observed on the $k_{h}$ th sensor of the $h$ th array becomes

$$
\Delta \phi_{k_{h}}=\exp \left(j 2 \pi \frac{r_{h}}{\lambda} \sin \theta \cos \left(\phi-\phi_{k_{h}}\right)\right)
$$

The steering vector of the $i$ th source signal on the $h$ th circular array in terms of the azimuth and elevation angles and also the position vectors of the sensor elements is as follows:

$$
\mathbf{a}_{k}\left(\theta_{i}, \phi_{i}\right)=\left[\begin{array}{c}
\exp \left(j 2 \pi \widehat{r}_{h} \sin \theta_{i} \cos \left(\phi_{i}-\phi_{0}\right)\right) \\
\exp \left(j 2 \pi \widehat{r}_{h} \sin \theta_{i} \cos \left(\phi_{i}-\phi_{1}\right)\right) \\
\vdots \\
\exp \left(j 2 \pi \widehat{r}_{h} \sin \theta_{i} \cos \left(\phi_{i}-\phi_{K_{h}-1}\right)\right)
\end{array}\right],
$$

where $\hat{r}_{h}$ is the normalized radius of the circle by the wavelength. Given $\theta=90^{\circ}$, the steering matrix of the $h$ th circular array becomes

$$
\begin{aligned}
& \mathbf{A}_{k}\left(\phi_{i}\right) \\
& =\left[\begin{array}{ccc}
e^{j 2 \pi \widehat{r}_{h} \cos \phi_{1}} & \cdots & e^{j 2 \pi \widehat{r}_{h} \cos \phi_{P}} \\
e^{j 2 \pi \widehat{r}_{h} \cos \left(\phi_{1}-\left(1 / K_{h}\right) 2 \pi\right)} & \cdots & e^{j 2 \pi \widehat{r}_{h} \cos \left(\phi_{P}-\left(1 / K_{h}\right) 2 \pi\right)} \\
\vdots & \vdots & \vdots \\
e^{j 2 \pi \widehat{r}_{h} \cos \left(\phi_{1}-\left(\left(K_{h}-1\right) / K_{h}\right) 2 \pi\right)} & \cdots & e^{j 2 \pi \widehat{r}_{h} \cos \left(\phi_{P}-\left(\left(K_{h}-1\right) / K_{h}\right) 2 \pi\right)}
\end{array}\right] .
\end{aligned}
$$

As the steering vector of a signal is equal to the multiplication of the slowness vector $\alpha$ and the position vector $\mathbf{r}$, the steering matrix $\mathbf{A}$ of CCA can be defined as a combination of the steering matrices of each individual UCA by taking the center of the arrays as the reference:

$$
\mathbf{A}(\theta, \phi)=\left[\begin{array}{c}
\mathbf{A}_{1} \\
\mathbf{A}_{2} \\
\vdots \\
\mathbf{A}_{H}
\end{array}\right]
$$


In case of $P$ source signals and a total of $M=\left(K_{1}+K_{2}+\right.$ $\left.\cdots+K_{H}\right)$ sensor elements, the observed signal model of CCA remains similar to the one of UCA in (1).

3.2. The Steering Matrix of a Concentric Circular Array. By applying implicit form of (17) to (18), we express the steering matrix of CCA as a combination of the steering vectors of each UCA:

$$
\mathbf{A}(\theta, \phi)=\left[\begin{array}{cccc}
\mathbf{a}_{0}\left(\theta_{1}, \phi_{1}\right) & \mathbf{a}_{0}\left(\theta_{2}, \phi_{2}\right) & \cdots & \mathbf{a}_{0}\left(\theta_{P}, \phi_{P}\right) \\
\mathbf{a}_{1}\left(\theta_{1}, \phi_{1}\right) & \mathbf{a}_{1}\left(\theta_{2}, \phi_{2}\right) & \cdots & \mathbf{a}_{1}\left(\theta_{P}, \phi_{P}\right) \\
\vdots & \vdots & \vdots & \vdots \\
\mathbf{a}_{H}\left(\theta_{1}, \phi_{1}\right) & \mathbf{a}_{H}\left(\theta_{2}, \phi_{2}\right) & \cdots & \mathbf{a}_{H}\left(\theta_{P}, \phi_{P}\right)
\end{array}\right] .
$$

If $\theta_{i}=90^{\circ}$ in the explicit form of the steering vectors, then the steering matrix $\mathbf{A}(\phi)$ becomes

$\mathbf{A}(\phi)$

$$
=\left[\begin{array}{ccc}
e^{j 2 \pi \widehat{r}_{1} \cos \phi_{1}} & \cdots & e^{j 2 \pi \widehat{r}_{1} \cos \phi_{P}} \\
e^{j 2 \pi \hat{r}_{1} \cos \left(\phi_{1}-\left(1 / K_{1}\right) 2 \pi\right)} & \cdots & e^{j 2 \pi \widehat{r}_{1} \cos \left(\phi_{P}-\left(1 / K_{1}\right) 2 \pi\right)} \\
\vdots & \vdots & \vdots \\
e^{j 2 \pi \widehat{r}_{1} \cos \left(\phi_{1}-\left(\left(K_{1}-1\right) / K_{1}\right) 2 \pi\right)} & \cdots & e^{j 2 \pi \widehat{r}_{1} \cos \left(\phi_{P}-\left(\left(K_{1}-1\right) / K_{1}\right) 2 \pi\right)} \\
\vdots & \vdots & \vdots \\
e^{j 2 \pi \widehat{r}_{H} \cos \left(\phi_{1}\right)} & \cdots & e^{j 2 \pi \widehat{r}_{H} \cos \left(\phi_{P}\right)} \\
e^{j 2 \pi \widehat{r}_{H} \cos \left(\phi_{1}-\left(1 / K_{H}\right) 2 \pi\right)} & \cdots & e^{j 2 \pi \widehat{r}_{H} \cos \left(\phi_{P}-\left(1 / K_{H}\right) 2 \pi\right)} \\
\vdots & \vdots & \vdots \\
e^{j 2 \pi \widehat{r}_{H} \cos \left(\phi_{1}-\left(\left(K_{H}-1\right) / K_{H}\right) 2 \pi\right)} & \cdots & e^{j 2 \pi \widehat{r}_{H} \cos \left(\phi_{P}-\left(\left(K_{H}-1\right) / K_{H}\right) 2 \pi\right)}
\end{array}\right] .
$$

\section{Simulations}

The simulations are developed in three aspects to observe the behavior of CCAs compared to UCAs in the DOA estimation. These are the effect of SNR, angle resolution, and the number of source signals, respectively.

4.1. Effect of SNR and Angle Resolution in DOA Estimation. In this section, $P_{\text {MUSIC }}(\theta, \phi)$ spatial spectrum graphics are generated for the same CCA geometry at different SNRs and DOAs of source signals. The noise is modeled as an additive white Gaussian noise (AWGN). The estimated DOAs are compared to the given DOA values for each scenario and the accuracies of the estimations are evaluated. There are two concentric rings $(H=2)$ each having 10 elements. The distance between two adjacent elements of the first circular array is $d=\lambda / 2$ and therefore $r_{0}=0.8090 \lambda$, whereas in the second array for $d=\lambda / 4$, the radius $r_{1}$ becomes $0.4045 \lambda$.

Scenario 1 . Let the elevation and the azimuth angles of the signals be

$$
\theta=\left(35^{\circ}, 45^{\circ}, 55^{\circ}\right), \quad \phi=\left(40^{\circ}, 50^{\circ}, 60^{\circ}\right),
$$

and the SNR of each source signal is $20 \mathrm{~dB}$. $P_{\text {MUSIC }}$ spatial spectrum contour plot of the aforementioned CCA geometry is given in Figure 3(a). In the scenario as the DOA difference

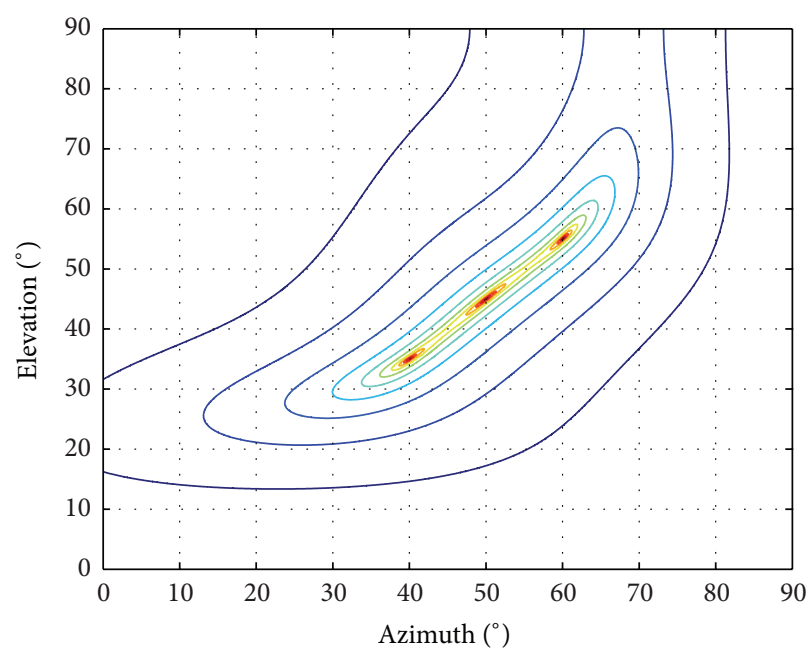

(a)

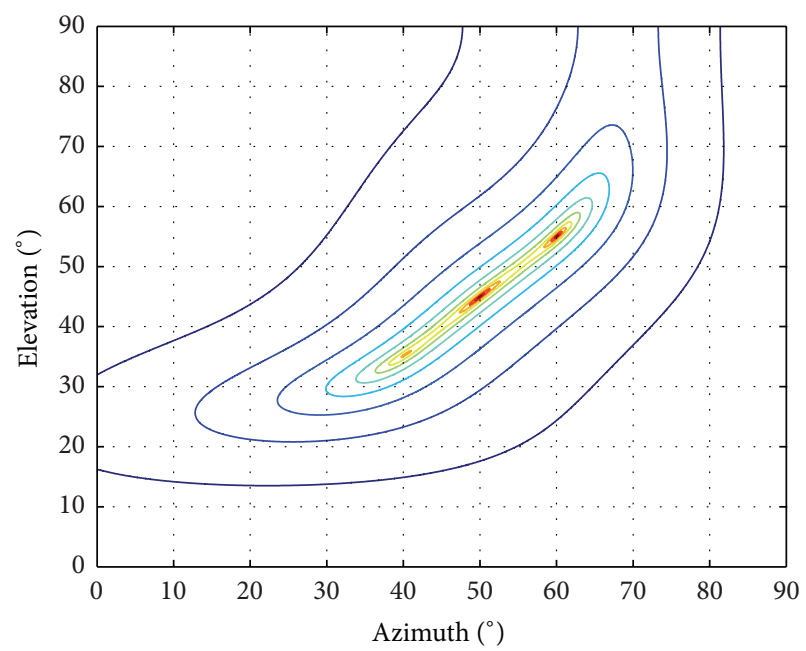

(b)

Figure 3: $P_{\text {MUSIC }}$ spatial spectrum of (a) Scenario 1 and (b) Scenario 2 in $\mathrm{dB}$ scale.

among source signals is $10^{\circ}$, which is sufficiently large and moreover the SNR values are high, the DOA estimations through $P_{\text {MUSIC }}$ spectrum are very accurate.

Scenario 2. Apart from Scenario 1, the SNR of $s_{1}(t)$ is chosen as $5 \mathrm{~dB}$ and this way a far (or a low-power) source signal is modeled. As the SNR decreases, the peak value of the corresponding source signal decreases as shown in Figure 3(b) which makes DOA estimation much more difficult.

Scenario 3. In this scenario the SNR values of all source signals are decreased up to $5 \mathrm{db}$ and the simulations are repeated under such a noisy medium keeping the DOA differences constant. As shown in Figure 4, the $P_{\text {MUSIC }}$ spatial spectrum contour plot is deteriorated and the probability of error in DOA estimation is increased because of low SNR values.

Scenario 4. To monitor the effect of decreased DOA differences, we analyze the situation where the angle difference 


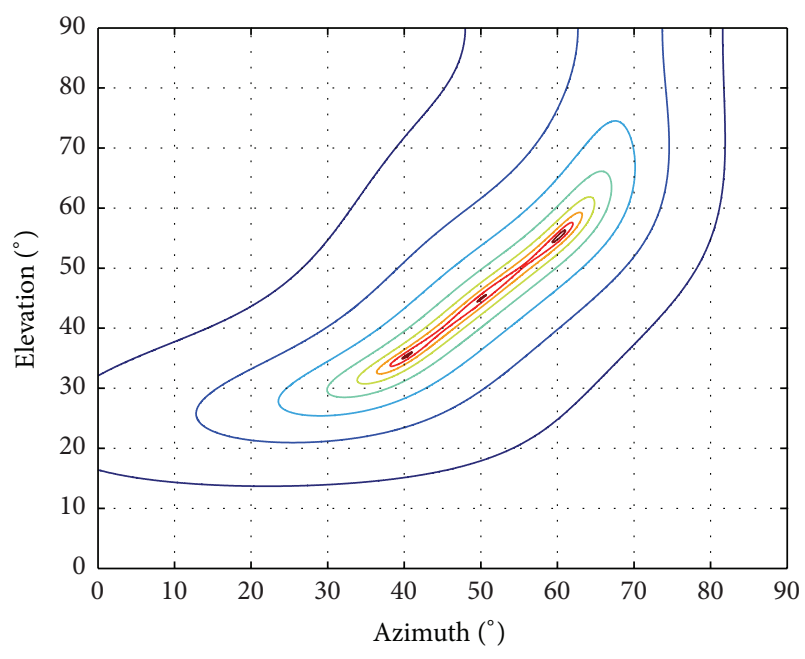

Figure 4: $P_{\text {MUSIC }}$ spatial spectrum of Scenario 3 in $\mathrm{dB}$ scale.

between source signals is only $2^{\circ}$; hence, the DOAs are very close to each other for three source signals. At first, SNR values are kept at $20 \mathrm{~dB}$ for each of them to compare the results to previous scenarios. The given DOAs are as follows:

$$
\theta=\left(35^{\circ}, 45^{\circ}, 55^{\circ}\right), \quad \phi=\left(38^{\circ}, 40^{\circ}, 42^{\circ}\right) \text {. }
$$

Since the SNR values are sufficiently high, it is possible to differentiate the signals and accurately estimate the DOAs with high resolution as presented in Figure 5(a). However, if the same scenario is repeated except the noise power with $\mathrm{SNR}=5 \mathrm{~dB}$, it is impossible to estimate the DOAs of the source signals as shown in Figure 5(b).

All of the scenarios provide a comparison of the MUSIC algorithm for various SNR values and resolution properties of the source signals in case of CCAs. Section 4.2 presents an intensive performance analysis of CCA with respect to other antenna array structures.

4.2. Performance Analysis of CCA. In this section, the DOA estimation performance of CCAs are investigated for various numbers of sensor elements, SNR values, and angle resolutions by MUSIC algorithm. In the scenarios, Monte-Carlo simulations are realized for all SNR values. The sensor arrays have a total of 7 or 10 elements and for each array geometry SNRs are varied from $5 \mathrm{~dB}, 10 \mathrm{~dB}, 15 \mathrm{~dB}$ to $20 \mathrm{~dB}$ and root mean square error (RMSE) values of the estimations are computed with respect to the given DOA values. The CCA with 7 elements includes two concentric rings with 3 and 4 array elements on each ring, whereas the 10-element arrays have 6 and 4 elements on each ring. In the first ring of the 7element CCA three sensors are used. The distance between two adjacent elements are chosen as $d=\lambda / 2$; therefore, the radius of the ring becomes $r_{0}=0.1443 \lambda$. The second concentric ring consists of four equispaced sensors where the distance among elements is chosen as $d=\lambda / 4$ which results in a radius of $r_{1}=0.3536 \lambda$. In comparison to CCAs, the UCA schemes contain 7 and 10 elements of $\lambda / 2$ distance in between, and therefore the radius of the UCAs is $0.5762 \lambda$ and $0.8090 \lambda$, respectively.

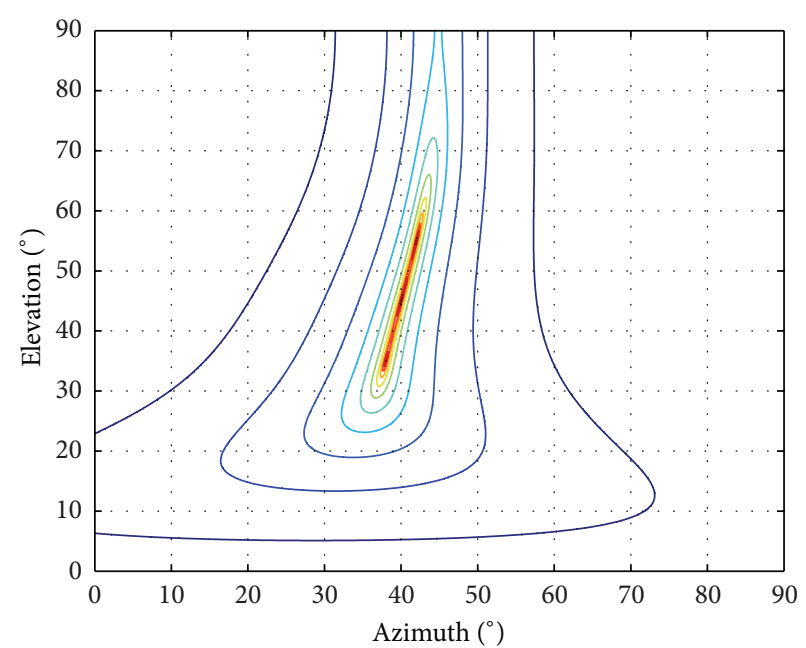

(a)

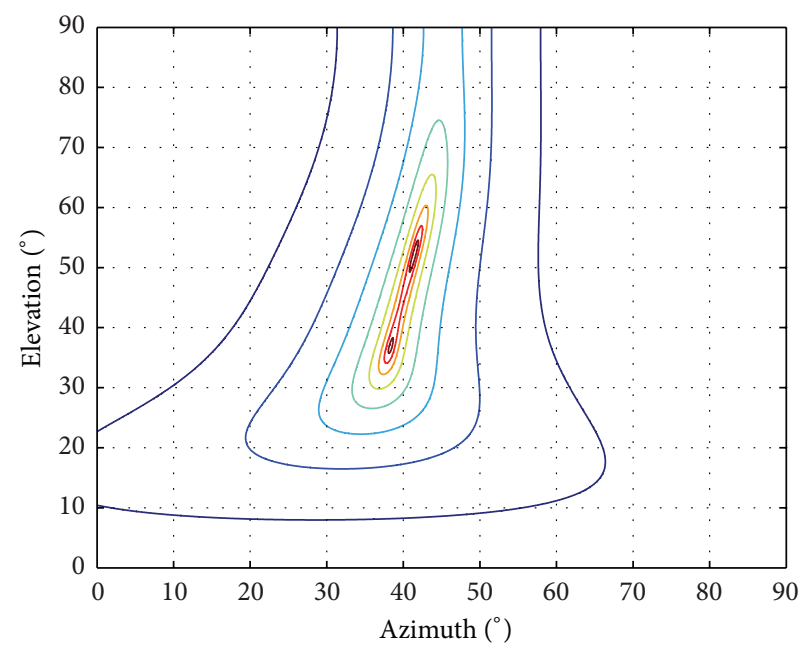

(b)

Figure 5: $P_{\text {MUSIC }}$ spatial spectrum of Scenario 4 (a) for $20 \mathrm{~dB}$ SNR, (b) for $5 \mathrm{db}$ SNR in $\mathrm{dB}$ scale.

4.2.1. Angle Resolution and SNR Analysis of Different CCAs. To analyze the change in the RMSE value with respect to the angle resolution for a fixed SNR, Monte-Carlo simulations are performed for three distinct source signals where the azimuth angle differences are $5^{\circ}, 10^{\circ}$, and $20^{\circ}$ at four different SNR values. The results of the CCA and UCA geometries are presented in Figures 6, 7, and 8. As shown in the figures CCA has better performance than UCA furthermore, increasing the number of elements in the arrays decreases the RMSE and makes the error ratios closer. In parallel, as the angle differences of the source signals increase, the performance in DOA estimation also increases. Increased SNR values provide better estimations, which approve the results presented in Section 4.1.

4.2.2. Varying the Number of Source Signals in DOA Estimation. To analyze the accuracy in DOA estimation with respect to the number of source signals, six different scenarios are considered for the CCA geometry of 7 sensor elements. 

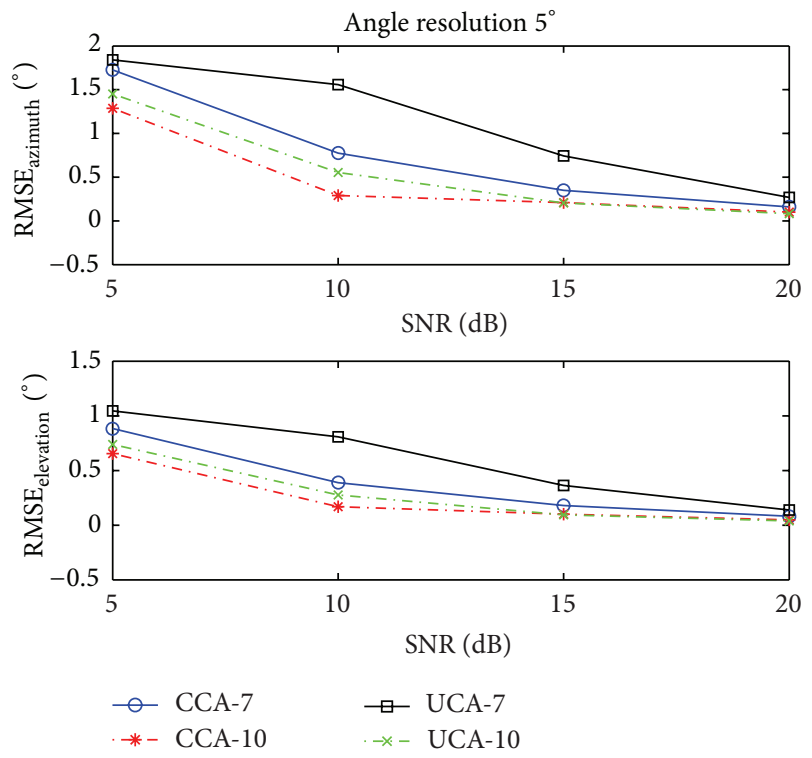

FIGURE 6: RMSEs for angle differences of $5^{\circ}$ among source signals.
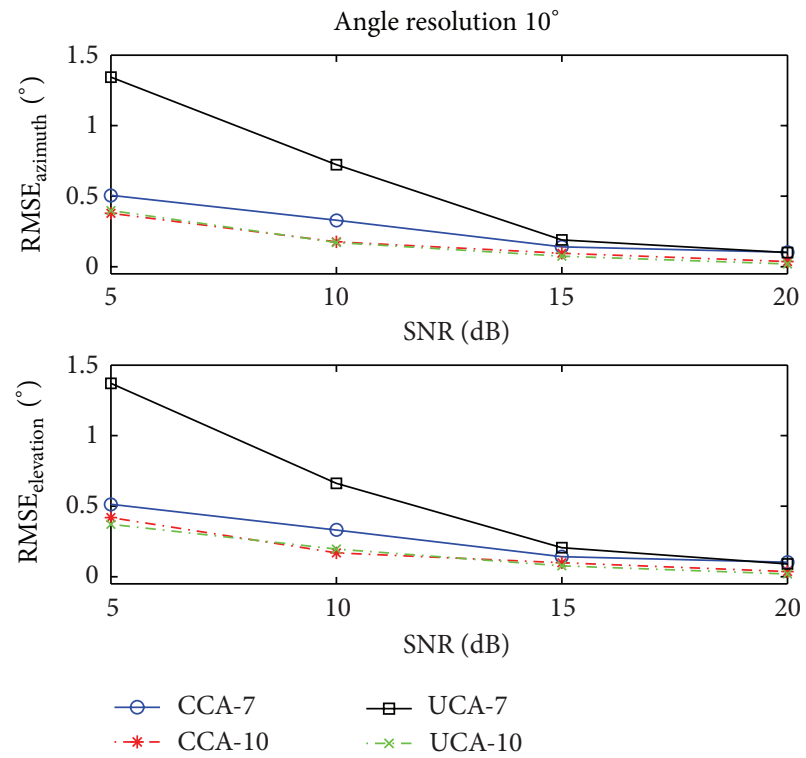

FigURE 7: RMSEs for angle differences of $10^{\circ}$ among source signals.

The number of source signals is varied from 1 to 6 and $P_{\text {MUSIC }}$ spatial spectrums are analyzed accordingly. The simulations are performed for $20 \mathrm{~dB}$ SNRs. Although the elevation angles are estimated in all of the simulations, for a clear representation of results, only azimuth angles are shown. In case of the single-source signal case, the DOA of the signal is chosen as $\left(\theta_{1}, \phi_{1}\right)=\left(90^{\circ}, 5^{\circ}\right)$. The resultant spectrum is presented in Figure 9(a). When the number of source signals are 2, as shown in Figure 9(b), DOA estimation is also successful. In this case, the DOAs of the source signals were chosen as $\left(\theta_{1}, \phi_{1}\right)=\left(90^{\circ},-15^{\circ}\right)$ and $\left(\theta_{2}, \phi_{2}\right)=\left(90^{\circ}, 15^{\circ}\right)$, respectively.

When the DOAs of signals are chosen as $\left(\theta_{1}, \phi_{1}\right)=$ $\left(90^{\circ},-35^{\circ}\right),\left(\theta_{2}, \phi_{2}\right)=\left(90^{\circ}, 5^{\circ}\right)$ versus $\left(\theta_{3}, \phi_{3}\right)=\left(90^{\circ}, 35^{\circ}\right)$, as shown in Figure 9(c), the estimation by the CCA is similar
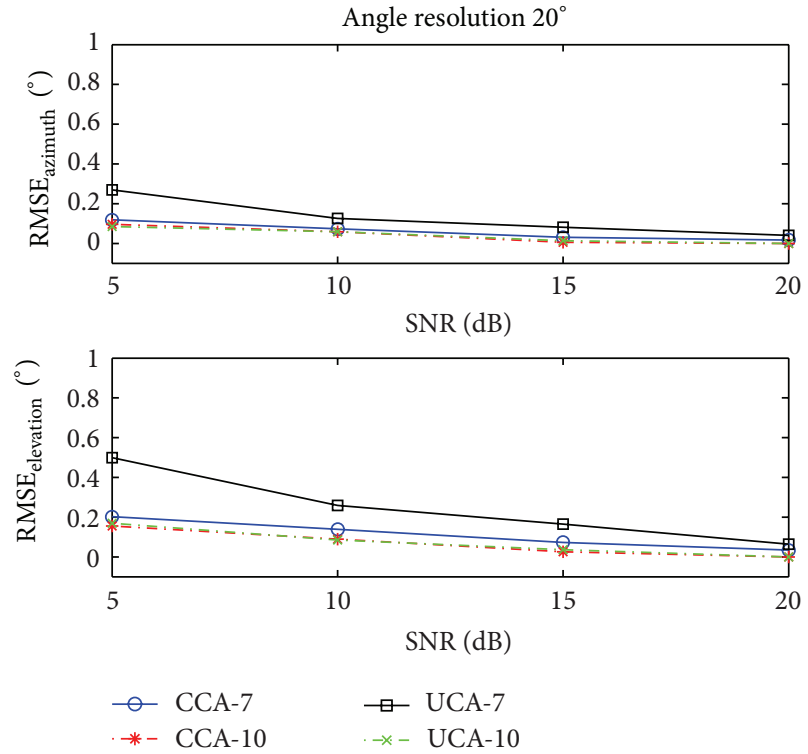

FIgURE 8: RMSEs for angle differences of $20^{\circ}$ among source signals.

to the exact values of the three source signals. When the number of source signals is increased up to 4 , and the DOAs are chosen as

$$
\theta_{\mathbf{i}}=90^{\circ} \quad \mathbf{i}=1, \ldots, 4, \quad \phi=\left(-45^{\circ},-15^{\circ}, 15^{\circ}, 45^{\circ}\right) .
$$

The 7-element CCA still can resolve the source signals, but as seen from Figure 9(d) the amplitudes of the peak values of the $P_{\text {MUSIC }}$ spectrum have decreased significantly. In case of a low-SNR situation, the algorithm may not estimate the DOAs accurately. If the number of source signals is increased up to 5 and 6 as shown in the spectrum plots in Figures 10(a) and 10(b), it is difficult to estimate the DOAs with a 7-element CCA. In the 5-source signal case, the DOAs of the source signals are chosen as

$$
\begin{aligned}
& \theta_{\mathbf{i}}=90^{\circ} \quad \mathbf{i}=1, \ldots, 5, \\
& \phi=\left(-65^{\circ},-35^{\circ}, 5^{\circ}, 35^{\circ}, 65^{\circ}\right) .
\end{aligned}
$$

We observe an extra peak at $\left(90^{\circ},-5^{\circ}\right)$. As this false peak is larger than the one belonging to $\left(\theta_{1}, \phi_{1}\right)=\left(90^{\circ},-65^{\circ}\right)$, the algorithm not only causes error in the DOA estimation values but also in the number of source signals. For 6 different source signals, with the following DOAs

$$
\begin{gathered}
\theta_{\mathbf{i}}=90^{\circ} \quad \mathbf{i}=1, \ldots, 6, \\
\phi=\left(-75^{\circ},-45^{\circ},-15^{\circ}, 15^{\circ}, 45^{\circ}, 75^{\circ}\right),
\end{gathered}
$$

it is impossible to estimate and differentiate source signals by a 7-sensor CCA.

\section{Conclusions}

Developing an original approach to the estimation of DOAs of target signals, we defined the DOA problem for antenna 


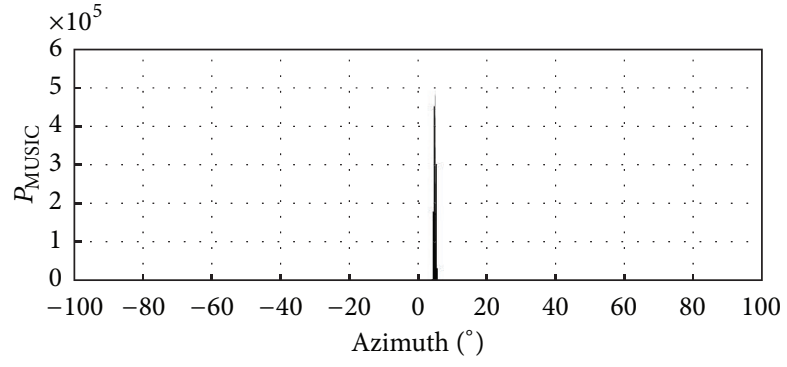

(a)

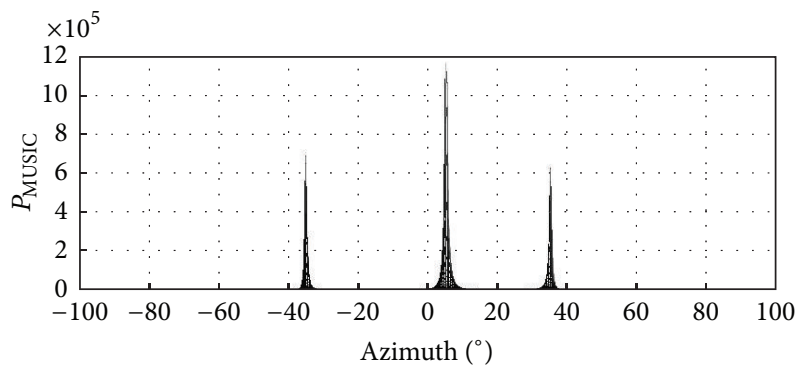

(c)

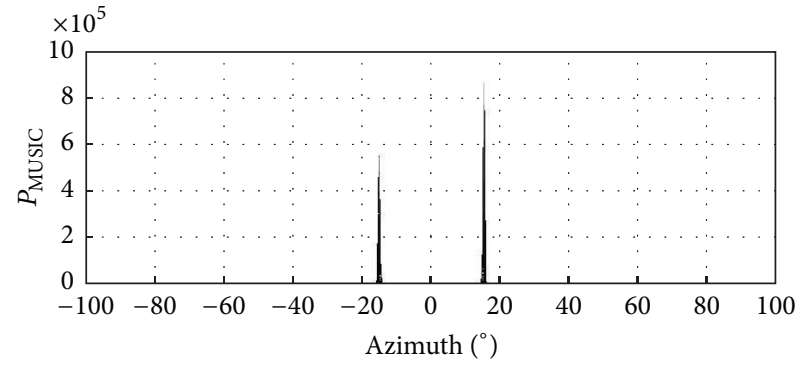

(b)

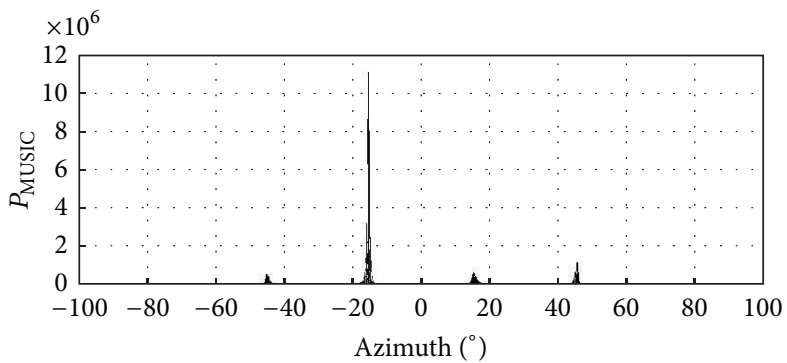

(d)

Figure 9: $P_{\text {MUSIC }}$ spectrums for (a) one, (b) two, (c) three, and (d) four source signals.

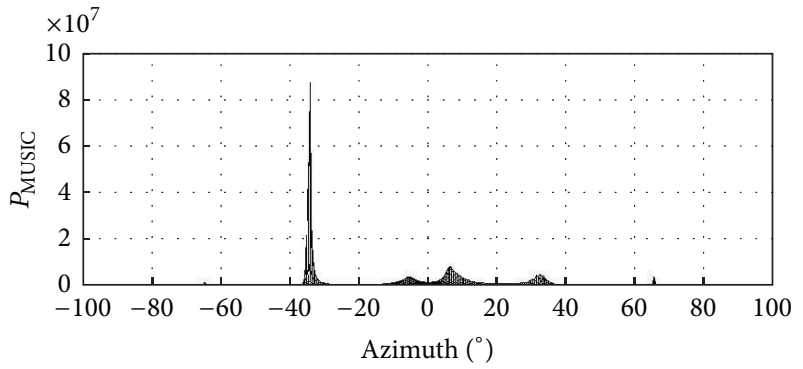

(a)

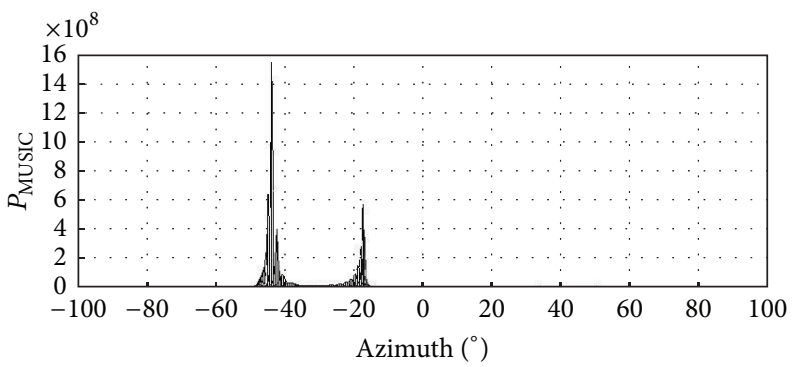

(b)

Figure 10: $P_{\text {MUSIC }}$ spectrums for (a) five and (b) six source signals.

arrays fitting in a concentric circular geometry and introduced the steering matrix of the CCA. We performed various scenarios to simulate the DOA estimation performance of CCAs. The results of simulations have been analyzed in terms of number of array elements and source signals, as well as SNR values and angle resolutions between the signals.

The simulations show that increasing SNR values and angle differences between the directions of incoming signals causes lower error rates in the DOA estimation. Similarly, increasing the number of antenna elements of CCA has a positive effect on the accuracy of estimations.

While ULAs can only estimate the azimuth angle, CCAs estimate both azimuth and elevation angles. For smaller angle differences among incoming signals, that is, for greater angle resolutions, the CCA provides more accurate estimation results than ULA. However, as the number of terms in the computational steps in the estimation algorithms of CCA and UCA is much more than ULAs, more computational power is needed to realize these algorithms. CCA can have less or similar error rates compared to UCA, for a given number of antennas. CCAs require smaller physical area than UCAs so this gives the CCA an important advantage to be a preferable alternative for using it in mobile applications.

Because of its multidimensional physical geometry, it is possible to generate a lot of scenarios to place the array elements in a CCA for a given number of antenna elements. As a result emanating from this physical realization diversity, it is harder but important to find the optimum solution compared to the ULA and UCA.

In future works, the physical locations of array elements in a CCA may be determined by developing optimization algorithms to achieve more reliable estimates of the parameters related to the DOA problem. This way, estimating accurate DOAs at low SNR values and differentiation of the source signals having small angle resolutions will be possible. Additionally another investigation issue with the CCA geometries will be on the estimation of DOAs of wide band signals.

\section{References}

[1] S. Chandran, Advances in Direction-of-Arrival Estimation, Artech House, Norwood, Mass, USA, 2006. 
[2] R. Fallahi and M. Roshandel, "Effect of mutual coupling and configuration of concentric circular array antenna on the signalto-interference performance in CDMA systems," Progress in Electromagnetics Research, vol. 76, pp. 427-447, 2007.

[3] E. D. di Claudio, "Asymptotically perfect wideband focusing of multiring circular arrays," IEEE Transactions on Signal Processing, vol. 53, no. 10, pp. 3661-3673, 2005.

[4] P. S. Naidu, Sensor Array Signal Processing, CRC Press, New York, NY, USA, 2001.

[5] M. Grice, J. Rodenkirch, A. Yakovlev, H. K. Hwang, Z. Aliyazicioglu, and A. Lee, "Direction of arrival estimation using advanced signal processing," in Proceedings of the 3rd International Conference on Recent Advances in Space Technologies (RAST '07), pp. 515-522, June 2007.

[6] R. O. Schmidt, "Multiple emitter location and signal parameter estimation," IEEE Transactions on Antennas and Propagation, vol. 34, no. 3, pp. 276-280, 1986.

[7] T. Wang, L. S. Yang, J. M. Lei, and S. Z. Yang, "A modified MUSIC to estimate DOA of the coherent narrowband sources based on UCA," in Proceedings of the International Conference on Communication Technology (ICCT '06), November 2006.

[8] K. R. Mahmoud, M. El-Adawy, S. M. M. Ibrahem, R. Bansal, and S. H. Zainud-Deen, "A comparison between circular and hexagonal array geometries for smart antenna systems using particle swarm optimization algorithm," Progress in Electromagnetics Research, vol. 72, pp. 75-90, 2007.

[9] F. Gozasht, G. R. Dadashzadeh, and S. Nikmehr, "A comprehensive performance study of circular and hexagonal array geometries in the lms algorithm for smart antenna applications," Progress in Electromagnetics Research, vol. 68, pp. 281296, 2007.

[10] M. Dessouky, H. Sharshar, and Y. Albagory, "Optimum normalized-Gaussian tapering window for side lobe reduction in uniform concentric circular arrays," Progress in Electromagnetics Research, vol. 69, pp. 35-46, 2007.

[11] M. Dessouky, H. Sharshar, and Y. Albagory, "Efficient sidelobe reduction technique for small-sized concentric circular arrays," Progress in Electromagnetics Research, vol. 65, pp. 187-200, 2006.

[12] S. C. Chan and H. H. Chen, "Uniform concentric circular arrays with frequency-invariant characteristics - theory, design, adaptive beamforming and DOA estimation," IEEE Transactions on Signal Processing, vol. 55, no. 1, pp. 165-177, 2007.

[13] S. C. Chan, H. H. Chen, and K. L. Ho, "Adaptive beamforming using frequency invariant uniform concentric circular arrays," IEEE Transactions on Circuits and Systems I, vol. 54, no. 9, pp. 1938-1949, 2007. 

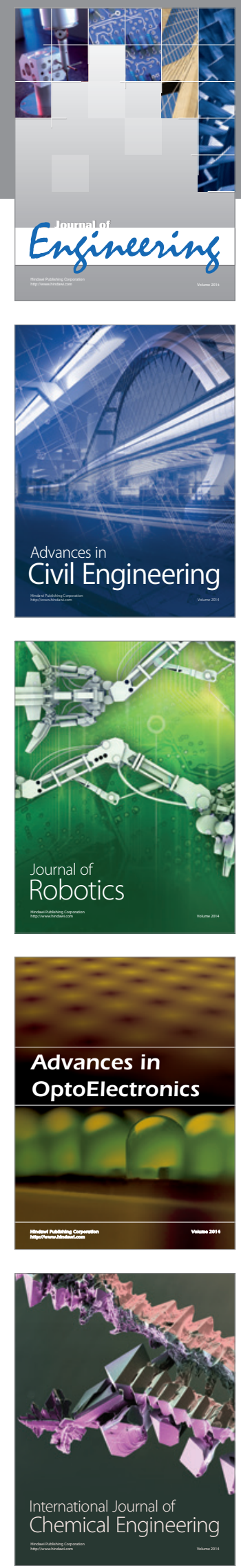

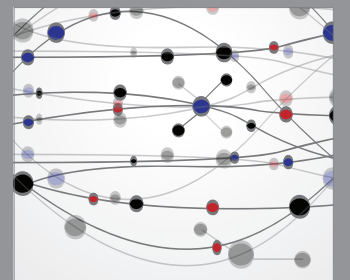

The Scientific World Journal
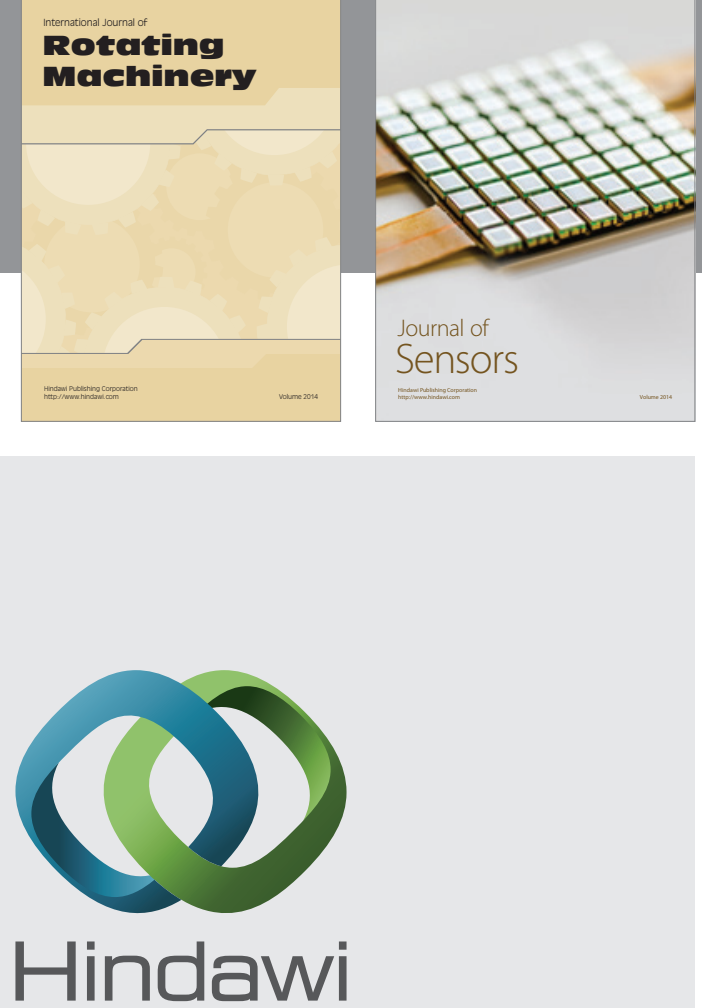

Submit your manuscripts at http://www.hindawi.com
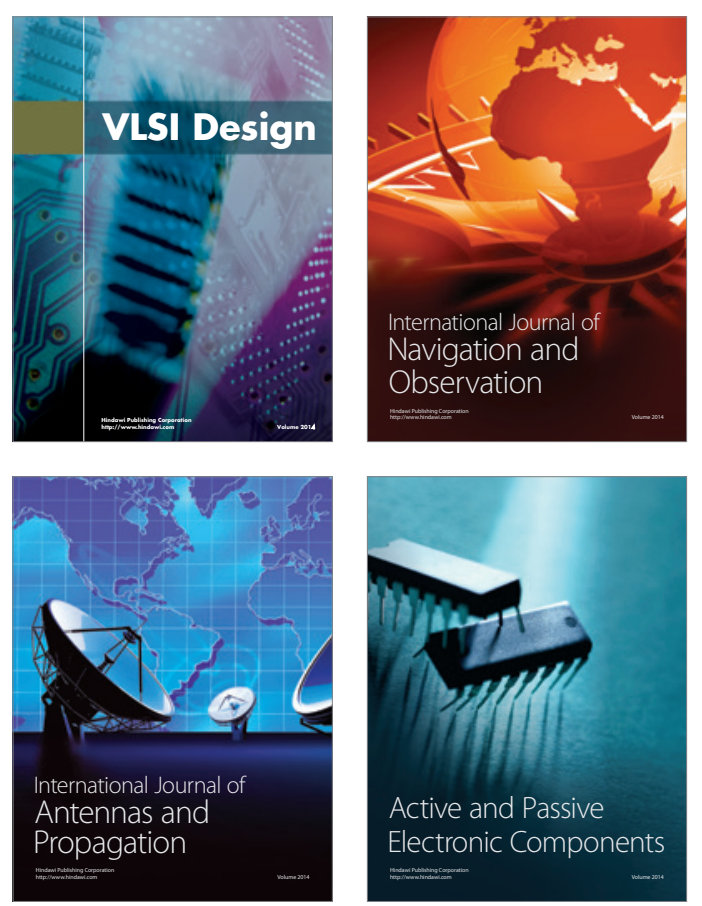
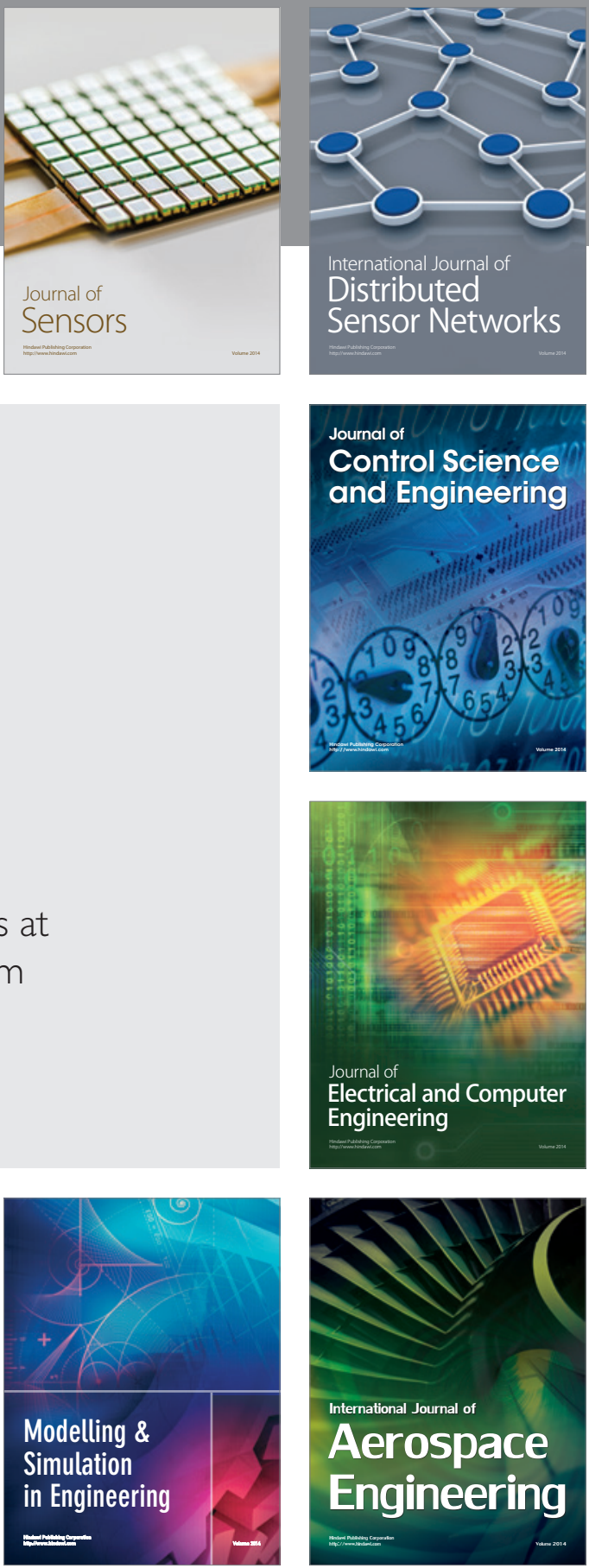

Journal of

Control Science

and Engineering
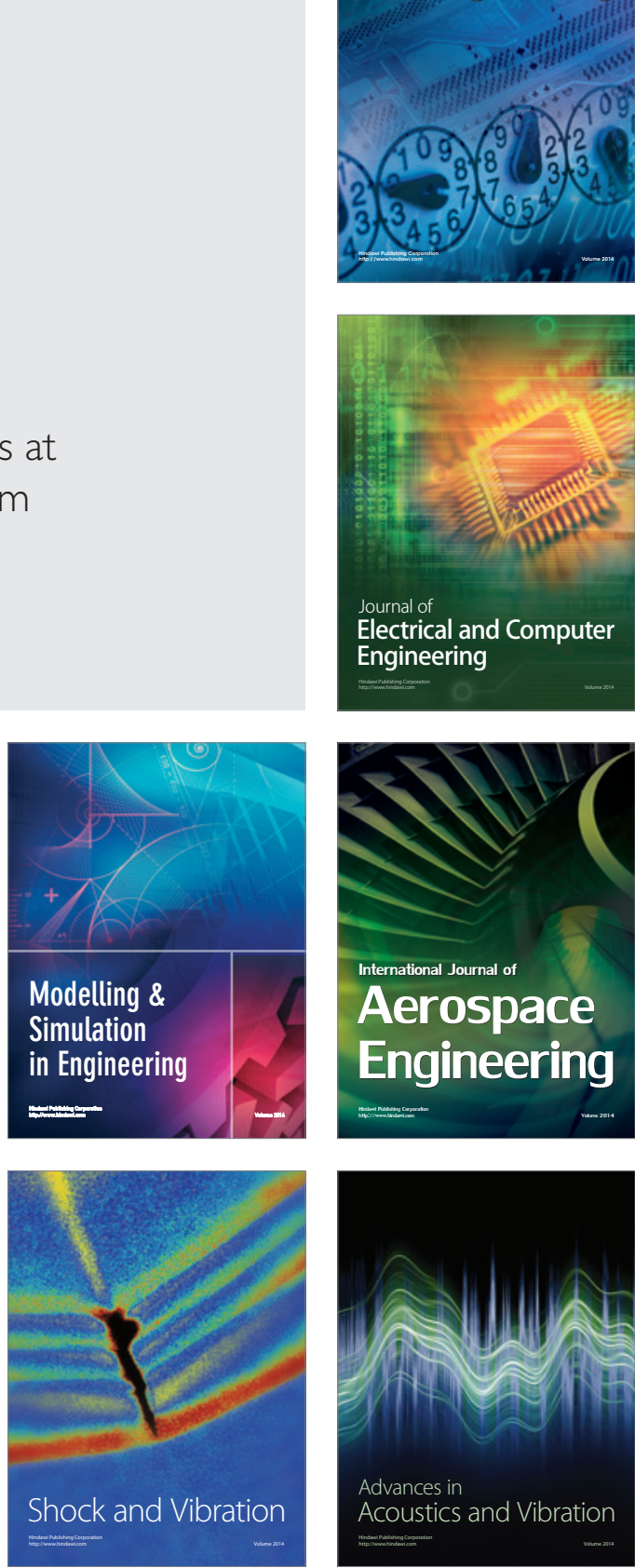